Available online at GSC Online Press Directory

GSC Biological and Pharmaceutical Sciences

e-ISSN: 2581-3250, CODEN (USA): GBPSC2

Journal homepage: https://www.gsconlinepress.com/journals/gscbps

(REVIEW ARTICLE)

\title{
Guillain-Barre syndrome
}

Raposo Anna Julia Lacerda 1, Freire Eduardo Galvão ${ }^{1}$, de Farias Célia Raiany Ferreira ${ }^{1}$, Serquiz e Pinheiro Renato 2, Pinheiro Francisco Irochima ${ }^{3}$, Guzen Fausto Pierdoná ${ }^{3}$, Rêgo Amália Cinhtia Meneses ${ }^{3}$ and Araújo-Filho Irami ${ }^{3, *}$

${ }^{1}$ Undergraduate Student of Medicine at UnP - Potiguar University - Laureate International Universities - Natal/Brazil.

${ }^{2}$ Neurologist and Professor at Potiguar University / UnP - Laureate International Universities - Natal/Brazil

${ }^{3}$ Postgraduate Program in Biotechnology at Potiguar University/ UnP - Laureate International Universities Natal/Brazil.

Publication history: Received on 19 October 2019; revised on 23 October 2019; accepted on 26 October 2019

Article DOI: https://doi.org/10.30574/gscbps.2019.9.1.0198

\begin{abstract}
Guillain-Barre syndrome is the most common and most severe acute flaccid paralysis, usually preceded by an infection or immune stimulation. A literature review of the last five years in Pubmed, Scopus, Embase, and Web of Science databases was done. Data were collected from epidemiological studies using the keywords. Guillain-Barrè syndrome (GBS) is an acute, severe, and fulminant polyradiculoneuropathy. It has ascending muscle weakness, rapid evolution, the involvement of respiratory muscles with an evolution period of up to four weeks. It has the Classic and Variant forms of clinical presentation. Guillain-Barrè Syndrome is a rapidly progressive disease. The diagnosis is clinical but requires exams such as electroneuromyography and cerebrospinal fluid analysis, so that treatment can be started as soon as possible to improve prognosis and increase survival of affected patients.
\end{abstract}

Keywords: Guillain-Barre syndrome; Acute infectious polyneuritis; Acute autoimmune neuropathy; Acute inflammatory demyelinating polyneuropathy; Inflammatory polyneuropathy acute

\section{Introduction}

Guillain-Barré Syndrome (GBS) is the most significant cause of generalized flaccid paralysis in the world [1,2], with an annual incidence of 1-4 cases per 100,000 population and a peak between 20 and 40 years of age [3]. GBS is an autoimmune disease that primarily affects the myelin of the proximal portion of the peripheral nerves, either acute or subacute. Approximately $60 \%$ to $70 \%$ of GBS patients have some previous critical illness ( 1 to 3 weeks earlier), with Campylobacter jejuni infection is the most common (32\%), followed by cytomegalovirus (13\%), Epstein Barr virus $(10 \%)$ and other viral infections such as hepatitis A, B, and C virus, influenza, and human immunodeficiency virus (HIV) [4-6]. Other minor precipitating factors are surgical intervention, immunization, and pregnancy [7,8]. Most patients initially perceive the disease by a feeling of paresthesia in the distal extremities of the lower and then upper limbs. Low back or neuropathic leg pain can be seen in at least 50\% of cases [2]. Progressive weakness is the most noticeable sign to the patient, usually occurring in this order: lower limbs, arms, trunk, head, and neck. The intensity may range from mild weakness, which does not even motivate the search for medical attention in primary care [9], to the occurrence of complete quadriplegia requiring mechanical ventilation (MV) due to accessory respiratory muscle paralysis. Facial weakness occurs in half of the cases throughout the disease. Between 5\%-15\% of patients develop ophthalmic paresis and ptosis. Sphincterian function is often preserved, while the loss of myotatic reflexes can precede sensory symptoms even in poorly affected muscles. Autonomic instability is a common finding, eventually causing relevant arrhythmias $[1,6]$ but rarely persisting after two weeks [8]. The disease usually progresses for 2 to 4 weeks. At least 50\%-75\% of

\footnotetext{
${ }^{*}$ Corresponding author

E-mail address: irami.filho@uol.com.br
} 
patients are affected by the second week, $80 \%-92 \%$ by the third week, and $90 \%-94 \%$ by the fourth week $[6,10]$. Respiratory failure requiring MV occurs in up to $30 \%$ of patients in this phase. Progression of signs and symptoms for more than eight weeks precludes the diagnosis of GBS, thus suggesting chronic inflammatory demyelinating polyneuropathy (CIDP). After the progression phase, GBS goes on a plateau for several days or weeks, with the subsequent gradual recovery of motor function over several months. However, only $15 \%$ of patients will have no residual deficit within two years of disease onset, and 5\% to $10 \%$ will remain with disabling motor or sensory symptoms. Mortality in GBS patients is approximately $5 \%$ to $7 \%$, usually resulting from respiratory failure, aspiration pneumonia, pulmonary embolism, cardiac arrhythmias, and hospital sepsis [6,11]. Risk factors for a poor functional prognosis are age over 50, preceding diarrhea, abrupt onset of severe weakness (less than seven days), need for MV, and motor neural conduction potential amplitude less than $20 \%$ of the standard limit [6,12-14]. Motor prognosis is better in children because they require less ventilatory support and recover faster [6]. Recurrence of the episode can occur in up to $3 \%$ of cases, and there is no relationship with the treatment used in the acute phase, as believed [15]. The diagnosis is clinical but requires exams such as electroneuromyography and cerebrospinal fluid analysis, so that treatment can be started as soon as possible to improve prognosis and increase survival of affected patients $[4,5]$. In this sense, due to the severity of the disease, which can affect millions of people worldwide, the present study aimed to evaluate the primary evidence and results found in scientific studies about the definition, risk factors, epidemiology, clinical manifestations, diagnosis and treatment of Guillain-Barre syndrome.

\section{Material and methods}

The present study was a literature review of the last five years on the subject, in Pubmed, Scopus, Embase, and Web of Science databases. Data were collected from epidemiological studies using the keywords: Guillain-Barre syndrome, acute infectious polyneuritis, acute autoimmune neuropathy, acute inflammatory demyelinating polyneuropathy, and inflammatory polyneuropathy acute. The authors were based on the following guiding question: "What is the main evidence and results found in scientific studies on the definition, risk factors, epidemiology, clinical manifestations, diagnosis and treatment of Guillain-Barre syndrome in the last five years?"

\section{Results and discussion}

\subsection{Definition}

Guillain-Barre syndrome (GBS) is a severe and fulminant acute polyradiculoneuropathy, characterized by ascending muscle weakness, rapid evolution within four weeks, and progressive involvement of respiratory muscles [6, 7].

\subsection{Epidemiology}

GBS has a global incidence of 1-2 cases / 100,000 / year. It is the most common cause of acute flaccid paralysis [8-10]. Although all age groups are affected, the incidence increases by approximately $20 \%$ every ten years from the first decade of life. The incidence is higher in males, and western countries, adults, are the most affected $[11,12]$.

\subsection{Etiology}

GBS is believed to result from an immune response to the previous infection by cross-reaction with the peripheral nerve due to molecular mimicry. In about two-thirds of cases, there is an acute infectious disease that is referred to within one to four weeks, usually a flu process or acute gastroenterocolitis. The immune response may be directed to the peripheral nerve myelin or axon, resulting in demyelinating and axonal forms of GBS [13-15]. Campylobacter jejuni infection is the most common precipitating factor present in $45-76 \%$ of cases. Cytomegalovirus, Epstein-Barr, Human Immunodeficiency Virus (HIV), and Zika virus are also associated with the onset of GBS [16,17]. A small percentage of patients develop GBS after immunization, surgery, trauma, or bone marrow transplantation [18].

\subsection{Clinical condition}

The cardinal clinical features of Guillain-Barre syndrome (GBS) are: rapidly progressive, ascending muscle weakness, starting in the lower limbs and progressing to the upper limbs and face; poorly delimited distal sensory disorder in the limbs, accompanied by absent or reduced deep tendon reflexes [3,4]. Neurological symptoms reach the maximum degree of disability within a range of hours to four weeks. After this period, there is a tendency for recovery, which is satisfactory in approximately $85 \%$ of cases $[8,9,14]$. Muscle weakness can range from mild difficulty walking to almost complete paralysis of all extremity muscles, and in some patients may affect the facial, respiratory, and bulbar muscles [5]. Because it is a heterogeneous disease, SBG is divided into subtypes, and through electrophysiological study we have the demyelinating form (AIDP), which is the most common; and the pure motor axonal (AMAN - Acute Motor Axial 
Neuropathy) and acute motor-sensory (AMSAN) motor forms, the most severe of the phenotypes, with rapid onset of complete paralysis $[15,18]$. Autonomic dysfunction is a well-known feature of GBS and a significant source of mortality. Dysautonomy has already been described in $2 / 3$ cases; however this number is not precise in current clinical practice. Presents tachycardia (most common), urinary retention, alternating hypertension with hypotension, orthostatic hypotension, bradycardia, arrhythmias, paralytic ileus, and sweating $[6,19]$. These signs usually disappear in the GBS plateau phase before motor improvement [19]. The installation mode of neurological signs and symptoms allows classifying GBS in Classic and Variant [4-6].

\subsubsection{Classical syndrome}

In this form, a neurological deficit occurs in approximately 50\% of GBS cases. Its classic clinical picture is a motor and sensory symptoms that begin at the extremities of the lower limbs and rise symmetrically to the trunk, arms, and cranial nerves. Motor signs predominate over the sensitive ones, which are lighter, mainly affecting the deep type sensitivity, generating paresthesias and pain $[10,18]$. The predominant symptom is a weakness of varying intensity. Since onset, signs, and symptoms intensify and advance over the days, reaching the peak of muscle weakness within two weeks $50 \%$ of patients; $80 \%$ of cases in three weeks and $90 \%$ in four weeks $[3,18]$. After this phase, the patient with GBS may remain stable for two to four weeks, followed by spontaneous recovery. Most patients develop well, and only $10 \%$ of them develop neurological sequelae, which compromise ambulation [11-13,18].

\subsubsection{Variant syndromes}

The variant form is defined by the regional involvement of the peripheral nervous system, being divided into paraparesis, pharyngocervical brachial, and Miller Fisher syndrome (MFS) forms, the last two most frequente [9]. The pharyngeal-cervical-brachial variant represents approximately $14 \%$ of GBS cases. The motor deficit is restricted to the bulbar, cervical, and upper limb muscles, with muscle hyporeflexia or areflexia and absence of lower limb weakness [1]. SMF affects 5-10\% of patients, particularly in North America and Europe. Typically, it has acute ophthalmoplegia, ataxia, and hyporeflexia/areflexia, and only $25 \%$ of cases will have limb weakness. Peak deficit occurs within one week, and improvement begins after 15 days with complete recovery within six months $[16,18]$. Exclusively sensory, motor or autonomic forms are also part of GBS variants but are less frequent $[10,14]$. Disorders affecting the myoneural plaque are the primary differential diagnoses for SMF and pharyngo-cervical-brachial variant [12].

\section{Diagnosis}

The initial diagnosis of GBS is based on the clinical presentation. Cardinal clinical features are progressive, predominantly symmetrical muscle weakness, and absent or reduced deep tendon reflexes. Weakness can range from mild difficulty walking to almost complete paralysis of all extremity/limb, facial, respiratory, and bulbar muscles [6, 10]. The clinical diagnosis of GBS is supported by electroneuromyography and cerebrospinal fluid (CSF) analysis, which show typical abnormalities. Therefore, lumbar puncture and electrodiagnosis are required in all patients suspected of GBS. These tests are also useful for ruling out alternative diagnoses $[2,10,18]$.

\subsection{Complementary exams}

Cerebrospinal fluid (CSF) examination: the presence of proteinocytological dissociation (high protein level with little cellularity). Most cellularity is normal $\left(\leq 4\right.$ cells $\left./ \mathrm{mm}^{3}\right)$; Although pleocytosis occurs, if the number of cells $/ \mathrm{mm}^{3}$ is above 10, the concomitance of other diseases (HIV, Lyme disease, sarcoidosis, etc.) should be considered. Since CSF may include normal levels of cells and proteins, especially at the onset of presentation, its utility is questionable and is most appropriate in cases of diagnostic doubt [6,11, 18-20]. Electroneuromyography (ENMG): Demonstrates evidence of demyelination, allowing to confirm or clarify the injured anatomical site. In the early stages of AIDP, ENMG may be normal or show prolongation of $\mathrm{F}$ wave latency, prolongation of distal latency, temporal dispersion, blockage, and reduction of conduction velocity following the evolution of the clinical picture. In AMAN and AMSAN, however, the injury pattern is the marked reduction or even absence of motor action potentials, which may be associated with fibrillation potentials. Neuronal electrical studies have prognostic value, especially when repeated during the first five weeks $[6$, 14, 18]. Laboratory tests: Serum potassium, sodium, calcium, magnesium, phosphorus, creatine kinase levels, among others, serve for differential diagnosis [21]. Other tests are used as clinically suspected. HIV serology, Syphilis, Herpes virus family, Lyme disease, porphyrins, thyroid hormones, heavy metal dosing, evidence of systemic inflammatory activity, among others [21]. Computed Tomography and Magnetic Nuclear Resonance: exclude other spinal cord injuries $[16,17]$. 


\section{Treatment}

Regardless of the etiology, in every patient with acute flaccid paralysis with ongoing GBS0, the first step in clinical management is cardiopulmonary monitoring due to the risk of respiratory failure, bulbar muscle weakness, which leads to loss of natural airway protection (swallowing/ secretion clearance) and potentially severe cardiovascular involvement [11,17]. All patients should be admitted to the intensive care unit; however, the indication is absolute in cases of hemodynamic instability or motor deficit that may compromise pulmonary function/respiration (30\%) $[4,16,17]$. As autonomic dysfunction is joint in GBS, close monitoring of blood pressure, volume, and heart rate is essential in the clinical management of these patients and should be instituted at the time of hospital admission [17].

\subsection{Predictors of acute respiratory failure [17]}

- Time from disease onset to hospital admission less than seven days;

- Inability to cough;

- Inability to stand;

- Inability to lift elbows;

- Inability to lift the head;

- Increased liver enzyme;

- Presence of facial or bulbar weakness.

The following parameters warn of impending respiratory arrest so that in the presence of one major or two minor criteria, the onset of mechanical ventilation (MV) is indicated [17]:

\subsection{Major criteria}

- $\mathrm{PaCO}_{2}>48 \mathrm{mmHg}$

- $\mathrm{PaO}_{2}<56 \mathrm{mmHg}$

- Maximum inspiratory pressure $<30 \mathrm{cmH}_{2} \mathrm{O}$

- Maximum expiratory pressure $<40 \mathrm{cmH}_{2} \mathrm{O}$

- Forced vital capacity $<20 \mathrm{~mL} / \mathrm{kg}$

\subsection{Minor criteria}

- Inefficient cough

- Impaired swallowing

- Atelectasis

Tracheostomy should be considered after two to three weeks of MV and may be postponed for another week if there is evidence of improvement. General measures include prophylaxis for deep vein thrombosis with low molecular weight heparin and compression stockings until the patient can walk. Those restricted to bed should change their position frequently to avoid pressure ulcers. Physical therapy and occupational therapy, as well as psychological support, are essential [17-19]. Treatment with intravenous human immunoglobulin or plasmapheresis is considered to be a disease modifier and should be instituted in patients whose minimal disability is ambulation, having more significant therapeutic benefit when given preferentially in the first two to four weeks of the disease. However, it is recommended to start therapy if the patient has the walking ability but is not getting improvement after four weeks of symptom onset $[3 ; 8 ; 9 ; 14 ; 17]$. In cases of motor impairment of GBS, with the rapid evolution of early respiratory failure, therapeutic intervention with immunoglobulin or plasmapheresis is unquestionable, since it aims to avoid or reduce the time of mechanical ventilation and achieve rapid recovery. In mild cases (mild sensory or motor symptoms), in turn, prepared conduct is adopted, as the disease may progress to spontaneous recovery. $[3-5,8,9,16]$. The efficacy of plasmapheresis and intravenous human immunoglobulin is equivalent in patients with Guillain-Barrè syndrome, with no additional benefit from its associated use. Unresponsiveness or neurological deterioration occurs in $25 \%$ of cases in both treatments. The choice depends on local availability, patient and family preference, risk factors, and contraindications [8-10]. When both therapies are equally available, and there are no contraindications for either of them, we suggest treatment with intravenous human immunoglobulin because of its ease of administration and is considered the first line of treatment in most countries [3,6,21]. Plasma exchange is performed at $200-250 \mathrm{~mL} / \mathrm{kg}$, four to six sessions over eight to ten days, while intravenous immunoglobulin is administered at a dose of $400 \mathrm{mg} / \mathrm{kg} /$ day for five days [12,17]. Corticosteroid therapy is not recommended because it is not sufficient, although some studies have shown short-term therapeutic benefits when associated with intravenous human immunoglobulin [17,19-21]. Pharmacological treatment of pain, which is a complaint in about two-thirds of cases, cannot be forgotten. The therapeutic options are gabapentin, 
carbamazepine, and opioids such as morphine since simple analgesics or anti-inflammatory drugs are not effective in pain management of these patients $[3,15,20]$. Even after treatment, neurological conditions may continue to deteriorate. This reflects the natural history of the disease or a misdiagnosis. Thus, it is useful to confirm that the GBS diagnosis is correct before deciding on retreatment [17].

\section{Complications}

Although disease-modifying therapy has improved GBS, many patients remain sequelae, suffering from fatigue or chronic pain, making Guillain Barrè syndrome a pathology of high morbidity and mortality $[9,13,14]$. The primary complications of GBS include neurological sequelae that compromise ambulation in about 10\% of cases, neuropathic pain, affecting $40-50 \%$ of patients, requiring the use of anticonvulsants, tricyclic antidepressants and/or opioids in refractory cases. Mortality occurs in 3-8\% of cases and is mainly due to respiratory failure, dysautonomia, sepsis, or embolism [7]. The worst prognostic factors with prolonged length of stay and severe neurological sequelae: age over 70 years, acute seizures within seven days, severe neurological disability, low values of muscle action potential $(<20 \%$ reduction in distal motor amplitude average), need for ventilatory support and previous diarrheal disease [7,19-21].

\section{Conclusion}

In conclusion, the present review allowed us to define the main clinical characteristics of GBS. Rapidly progressive and ascending muscle weakness, a poorly delimited distal sensory disorder in the limbs, accompanied by absent or reduced deep tendon reflexes. The diagnosis of GBS is predominantly clinical. Electroneuromyography and cerebrospinal fluid analysis required for diagnostic definition. Treatment with intravenous human immunoglobulin or plasmapheresis in the first weeks offers a better prognosis. However, as it is a pathology of high morbidity, it deserves attention from health professionals for its early identification and proper management.

\section{Compliance with ethical standards}

\section{Acknowledgments}

The authors thank Prof. Renato Serquiz for the supervision of this review, acting as an expert consultant on the bibliographic survey, analysis, and scientific advice. We also thank all the study components for their dedication and effort to build a scientifically validated quality review.

\section{Disclosure of conflict of interest}

There are no conflicts of interest to declare by any of the authors of this study.

\section{References}

[1] Wakerley BR and Yuki N. (2014). Pharyngeal-cervical-brachial variant of Guillain-Barré syndrome. J Neurol Neurosurg Psychiatry, 85(3), 339-44.

[2] Beattie J, Parajuli S, Sanger M, Lee G, Pleninger P, Crowley G, Kwon S, Murthy V, Manko JA, Caplan A, Dufort E, Pastula DM and Nolan A. (2018). Zika Virus-Associated Guillain-Barré Syndrome in a Returning US Traveler. Infect Dis Clin Pract, 26(6), e80-e84.

[3] L Peña, Moreno CB and Gutierrez-Alvarez AM. (2015). Pain management in Guillain-Barre syndrome: A systematic review. Neurologia, 30(7), 433-438.

[4] Azevedo MB, Coutinho MSC, Silva MAD, Arduini DB, Lima JDV, Monteiro R, Mendes BNB, Lemos MCF, Noronha CP and Saraceni V. (2018). Neurologic manifestations in emerging arboviral diseases in Rio de Janeiro City, Brazil, 2015-2016. Rev Soc Bras Med Trop, 51(3), 347-351.

[5] Eldar AH and Chapman J. (2014). Guillain Barré syndrome and other immune mediated neuropathies: Diagnosis and classification. Autoimmunity Reviews, 13(4-5), 525-30.

[6] Wijdicks EF and Klein CJ. (2017). Guillain-Barré Syndrome. Mayo Clinic Proceedings, 92(3), 467-479.

[7] Edmundson C and Bird SJ. (2019). Acute Manifestations of Neuromuscular Disease. Thieme Medical Publishers, 39(1), 115-124. 
[8] Motamed-Gorji N, Matin N, Tabatabaie O, Pavone P, Romano C, Falsaperla R and Vitaliti G. (2017). Biological Drugs in Guillain-Barré Syndrome: An Update. Curr Neuropharmacol, 15(7), 938-950.

[9] Goodfellow JA and Willison HJ. (2016). Guillain-Barré syndrome: a century of progress. Nature Reviews Neurology, 12(12), 723-731.

[10] Willison HJ, Jacobs BC and Doorn PAV. (2016). Guillian-Barré syndrome. Lancet, 13, 388(10045), 717-727.

[11] Esposito S and Longo MR. (2017). Guillain-Barré syndrome. Autoimmun Rev, 16(1), 96-101.

[12] Kaushik R, Kharbanda PS, Bhalla A, Rajan R and Prabhakar S. (2014). Acute Flaccid paralysis in adults: Our experience. J Emerg Trauma Shock, 7(3), 149-54.

[13] Créange A. (2016). Guillain-Barré syndrome: 100 years on. Revue Neurologique, 172(12), 770-774.

[14] Jacobs BC, van den Berg B, Verboon C, Chavada G, Cornblath DR, Gorson KC, Harbo T, Hartung HP, Hughes RAC, Kusunoki S, van Doorn PA and Willison HJ; IGOS Consortium. (2017). International Guillain-Barré Syndrome Outcome Study: protocol of aprospective observational cohort study on clinical and biological predictors of disease course and outcome in Guillain-Barré syndrome. J Peripher Nerv Syst, 22(2), 68-76.

[15] Hughes RAC, Cornblath DR and Willison HJ. (2016) Guillain-Barré syndrome in the 100 years since its description by Guillain, Barré and Strohl. Brain, 139(11), 3041-3047.

[16] Jasti AK, Selmi C, Sarmiento-Monroy JC, Vega DA, Anaya JM and Gershwin ME. (2016). Guillain-Barré syndrome: causes, immunopathogenic mechanisms and treatment. Expert Rev Clin Immunol, 12(11), 1175-1189.

[17] Fadia M, Shroff S and Simpson E. (2019). Immune-Mediated Neuropathies. Curr Treat Options Neurol, $21(6), 28$.

[18] Bowley MP and Chad DA. (2019). Clinical neurophysiology of demyelinating polyneuropathy.Handbook of Clinical Neurology, 161(3), 241-268.

[19] Chakraborty T, Kramer CL, Wijdicks EFM and Rabinstein AA. (2019). Dysautonomia in Guillain-Barré Syndrome: Prevalence, Clinical Spectrum, and Outcomes. Neurocrit Care, 11.

[20] Doets AY, Jacobs BC and van Doorn PA. (2018). Advances in management of Guillain-Barré syndrome. Current Opinion in Neurology, 31(5), 541-550.

[21] Oaklander AL and Nolano M. (2019). Scientific Advances in and Clinical Approaches to Small-Fiber Polyneuropathy: A Review. JAMA Neurol.

\section{How to cite this article}

Raposo Anna JL, Freire EG, de Farias Célia RF, Serquiz e PR, Pinheiro FI, Guzen FP, Rêgo ACM and Araújo-Filho I. (2019). Guillain-Barre syndrome. GSC Biological and Pharmaceutical Sciences, 9(1), 98-103. 